\title{
Niveaux marins extrêmes en France : état des lieux
}

\author{
Franck Mazas, Luc Hamm
}

SOGREAH Maritime, Groupe ARTELIA. - 6 rue de Lorraine, 38130 Échirolles, France.

franck.mazas@sogreah.fr -luc.hamm@sogreah.fr

\begin{abstract}
RÉSUMÉ. - Un état des lieux des travaux sur la détermination des niveaux marins extrêmes au niveau centennal le long des différentes façades maritimes françaises métropolitaines est présenté. Dans un premier temps, les différentes méthodes statistiques utilisées pour les calculer sont rappelées, en distinguant les approches directes des approches indirectes. Dans un second temps, les résultats disponibles dans la littérature sont exposés pour les trois grandes façades maritimes (Manche et Mer du Nord, Atlantique, Méditerranée). Enfin, deux pistes d'amélioration sont esquissées, concernant d'une part l'approche régionale et d'autre part l'homogénéisation des données.
\end{abstract}

Mots-clés : niveaux extrêmes, France, maxima annuels, méthode du renouvellement, probabilité conjointe.

\section{Extreme sea levels in France : an overview}

\begin{abstract}
This paper presents a review of research works recently published in the literature related to the estimation of the 1-in-100-year extreme sea levels along French metropolitan coasts. Firstly, the different statistical methods used for these studies are summarized, with a clear distinction between the direct and indirect approaches combining storm surge and astronomical tidal levels. Secondly, available results are gathered and compared for the main three maritime frontages of France : North Sea / English Channel, Atlantic Ocean and Mediterranean Sea. Finally, two research topics are outlined for improving the current methodologies : namely the regional analysis approach and a special attention to data homogenization.
\end{abstract}

Key-words : extreme sea levels, France, annual maxima, Peak-over-threshold, joint probability methods.

\section{INTRODUCTION}

Les conséquences dévastatrices des submersions causées par la tempête Xynthia du 28 février 2010 ont rappelé l'importance cruciale de la prévision des niveaux marins extrêmes pour la protection du littoral et plus particulièrement de ses zones habitées. En plus de la nécessaire compréhension en profondeur de cet évènement singulier, c'est donc l'occasion de dresser un état des lieux le plus complet possible, non seulement des valeurs des niveaux extrêmes actuellement considérées le long des côtes françaises, mais également des acteurs impliqués et surtout des méthodes utilisées. Le présent papier vise donc, après un bref rappel sur les composantes d'un niveau marin (section II), à récapituler les diverses méthodes utilisées à l'heure actuelle (section III) ainsi qu'à rassembler les différents résultats obtenus par les équipes travaillant sur le sujet (section IV). Enfin, quelques perspectives d'amélioration sont avancées (section V).

\section{COMPOSANTES D'UN NIVEAU MARIN}

Le présent article se concentre sur le traitement des deux composantes les plus importantes d'un niveau d'eau : la marée astronomique et les surcotes / décotes météorologiques. Or, en pratique, on dispose généralement de séries temporelles de niveaux mesurés aux marégraphes. L'extraction de ces deux composantes peut se révéler ardue. En effet, le niveau de la mer subit l'influence de multiples phénomènes physiques et peut se décomposer comme suit :
- un niveau moyen, pouvant être défini à de multiples échelles de temps et subissant des fluctuations à ces mêmes échelles de temps : tendance à long terme due notamment au changement climatique, oscillations décadales (par exemple du fait des courants géostrophiques) et saisonnières ;

- la marée astronomique, phénomène déterministe pouvant être prédit localement à l'aide d'un nombre plus ou moins grand d'ondes élémentaires de marée ;

- les surcotes / décotes météorologiques, résultant de l'action $\mathrm{du}$ vent et de la pression (effets statique et dynamique) sur la surface libre ;

- les seiches côtières qui sont des ondes basse-fréquence de période allant de 10 à 50 minutes générées par de brusques variations météorologiques ;

- l'action des vagues, dont les fluctuations haute-fréquence sont filtrées par les marégraphes mais qui peuvent générer par déferlement sur les plages des ondes basse-fréquence et une surélévation du niveau moyen (set-up) ;

- des phénomènes exceptionnels comme les raz-de-marée (séismes, éruptions volcaniques, effondrements de terrain...) qui sont aisément repérables par datation.

La pratique actuelle [20] consiste d'abord à filtrer le signal pour en extraire un niveau moyen et à centrer le signal autour de cette moyenne. Puis, dans une seconde étape, à traiter le signal centré par une analyse harmonique ou spectrale pour en extraire le signal complet de la marée et en reconstituer un signal temporel. La dernière étape consiste à faire la différence entre le signal centré et le niveau de marée reconstitué qui constitue un résidu appelé compo- 
sante météorologique. Ce résidu englobe aussi bien les surcotes / décotes que les éventuelles seiches côtières ainsi que le set-up.

En pratique, la difficulté réside dans le nombre d'ondes de marée nécessaires pour extraire le signal complet de marée ainsi que dans le traitement de niveau moyen. Il est recommandé d'effectuer des calculs de niveau moyen à différentes périodes de filtrage pour identifier les différentes oscillations, et de retirer au moins la tendance à long terme. Beaucoup d'auteurs travaillent simplement sur la marée d'une part, et le résidu « niveau moins marée » d'autre part, englobant dans ce dernier l'ensemble des phénomènes cités ci-dessus. Il apparaît donc que le traitement des fluctuations du niveau moyen doit faire l'objet d'une grande attention dans les études sur les niveaux.

\section{LES MÉTHODES DE DÉTERMINATION DES NIVEAUX EXTRÊMES}

\section{III.1. Importance relative de la surcote météorologique et de la marée astronomique}

En première approche, le niveau de la mer est donc l'addition de la marée astronomique (composante déterministe) et de la composante météorologique (composante stochastique, c'est-à-dire aléatoire). La théorie statistique indique que ces deux composantes, de nature différente, doivent être traitées séparément. C'est ce que l'on appelle la méthode indirecte. Il est cependant possible de ne pas respecter cette indication et de traiter statistiquement le niveau d'eau dans son ensemble, sans effectuer cette séparation (c'est la méthode directe).

En pratique, l'importance relative d'une composante par rapport à l'autre peut être déterminante dans le choix de la méthode utilisée. Ainsi, l'étude récente de Haigh et al. (2010) [8] examine de manière systématique l'écart entre les deux approches. Elle met en évidence une similitude de résultats tant que le ratio marée/surcote est inférieur à 1,5 . (Ce ratio est défini comme le quotient du centile à $98 \%$ de la composante de marée, définie par rapport à un niveau moyen fixé à zéro, sur le centile à $98 \%$ des surcotes.) Au-delà de ce ratio, les méthodes directes sous-estiment (parfois fortement) les niveaux extrêmes par rapport aux méthodes indirectes.

A titre d'exemple, les surcotes maximales seront de l'ordre de 1 à $1,20 \mathrm{~m}$ sur environ tout le littoral français, alors que le marnage maximal peut varier de 13,42 $\mathrm{m}$ à Saint-Malo à seulement $0,40 \mathrm{~m}$ à Marseille. Suivant le site étudié, ce sera donc la composante déterministe ou la composante stochastique qui sera prédominante.

\section{III.2. Les méthodes directes}

\section{III.2.1. Description}

Le grand avantage des méthodes directes est leur simplicité. Leur inconvénient est cependant manifeste : la marée astronomique et la surcote météorologique ont un comportement très différent, surtout en ce qui concerne les extrêmes. Cependant, dans les cas où les marées sont très faibles par rapport aux surcotes, cette méthode peut fournir une bonne approximation (on pense notamment au littoral méditerranéen). Aussi détaille-t-on ici le principe des différentes méthodes directes existantes. De plus, ces traitements sont identiques à ceux appliqués à la seule variable stochastique (surcote) dans les méthodes indirectes.
On peut identifier quatre méthodes pour extraire de la série temporelle de départ les valeurs extrêmes à extrapoler : l'échantillon complet; les maxima annuels; les $r$ plus grandes valeurs ; la méthode POT. Chacun de ces pré-traitements va déterminer le modèle statistique à appliquer à l'échantillon résultant des valeurs extrêmes.

\section{III.2.2. La méthode de l'échantillon complet}

La méthode de l'échantillon complet consiste à utiliser toutes les données de la série temporelle pour ajuster la loi statistique qui semble la plus adaptée. Pour les marégraphes, il s'agit habituellement de mesures horaires, voire de mesures toutes les 15 minutes depuis une quinzaine d'années. L'inconvénient est double : d'une part, les données ne sont pas indépendantes entre elles (condition nécessaire à un bon ajustement statistique), et d'autre part, les queues de distributions, c'est-à-dire les valeurs extrêmes, sont mal ajustées, conduisant à des estimations imprécises des niveaux extrêmes. Cette méthode est donc systématiquement rejetée.

\section{III.2.3. La méthode des maxima annuels et le modèle GEV}

La méthode des maxima annuels consiste à retenir la valeur maximale de chaque année. Elle peut être étendue à n'importe quelle période (ou «bloc temporel »), par exemple en prenant les maxima mensuels. La loi naturelle de la méthode des maxima annuels est la distribution généralisée des valeurs extrêmes, ou loi GEV. En effet, étudions la loi de probabilité d'un échantillon d'une durée fixée, par exemple un an, de $n$ variables aléatoires réelles, indépendantes et identiquement distribuées suivant une distribution $F$. Cet échantillon s'écrit : $\left(X_{1}, \ldots, X_{n}\right)$. Cela peut être, par exemple, des mesures horaires ou tri-horaires. Soit $M_{n}$ le maximum de cet échantillon. La loi de probabilité, ou fonction de répartition, de $M_{n}$ s'écrit :

$\operatorname{Pr}\left\{M_{n} \leq x\right\}=\operatorname{Pr}\left\{X_{1} \leq x, X_{2} \leq x, \ldots, X_{n} \leq x\right\}=\prod_{i=1}^{n} \operatorname{Pr}\left\{X_{i} \leq x\right\}$

Soit :

$$
\operatorname{Pr}\left\{M_{n} \leq x\right\}=F^{n}(x)
$$

Le théorème des valeurs extrêmes stipule que lorsque $n$ tend vers l'infini, la loi de $M_{n}$ (via une renormalisation) tend vers l'une des trois familles de distributions suivantes : Gumbel, Fréchet ou Weibull. A partir de ce résultat, on peut combiner ces trois familles en un seul modèle, appelé la Distribution Généralisée des Valeurs Extrêmes, ou loi GEV :

$$
\operatorname{Pr}\left\{M_{n} \leq x\right\}=G_{x, k, \sigma, \mu}(x)=\exp \left[-\left(1+k \frac{x-\mu}{\sigma}\right)^{-\frac{1}{k}}\right]
$$

$k$, $\sigma$ et $\mu$ sont les paramètres de forme, d'échelle et de localisation de la distribution. Le premier détermine la forme fonctionnelle de la distribution, le deuxième régit sa dispersion et le dernier permet de fixer son origine. Lorsque $k \rightarrow 0$, la GEV se réduit à la loi de Gumbel définie par :

$$
G_{x, \sigma, \mu}(x)=\exp \left[-\exp \left(-\frac{x-\mu}{\sigma}\right)\right]
$$

L'avantage est qu'en construisant l'échantillon à partir des maxima annuels, ou même mensuels, on garantit l'indépendance des données. En revanche, la perte d'information est importante, puisque l'on ne sélectionne qu'une seule valeur par an (ou par mois). 


\section{III.2.4. La méthode des $r$ plus grandes valeurs}

Pour pallier ce problème, une extension de ce modèle a été construite en ne sélectionnant plus le seul maximum d'une année (ou de n'importe quel bloc temporel), mais les $r$ plus grandes valeurs : $\left(M_{n}^{(1)}, M_{n}^{(2)}, \ldots, M_{n}^{(r)}\right), M_{n}^{(1)}$ étant le maximum de la série temporelle au sein d'un bloc et $M_{n}^{(r)}$ la $r$-ième plus grande valeur.

Le théorème des valeurs extrêmes est généralisé pour obtenir la densité de probabilité conjointe suivante :

$$
\begin{aligned}
f\left(x^{(1)}, \ldots x^{(r)}\right)=\exp [-(1+ & \left.\left.k \frac{x^{(r)}-\mu}{\sigma}\right)^{-\frac{1}{k}}\right] \\
& \times \prod_{k=1}^{r} \frac{1}{\sigma}\left(1+k \frac{x^{(k)}-\mu}{\sigma}\right)^{-\frac{1}{k}-1}
\end{aligned}
$$

Cette loi ne dépend donc que de trois paramètres $(k, \sigma$ et $\mu$ ) à partir d'un échantillon $r$ fois plus étoffé que dans la méthode des maxima annuels, réduisant ainsi la variance de l'ajustement. En revanche, il demeure une certaine perte d'information sur les valeurs extrêmes de l'échantillon : si, par exemple, on fixe $r=5$, la $8^{\text {ème }}$ plus grande donnée d'une année agitée pourra être plus grande que la $5^{\text {ème }}$ plus grande donnée d'une année calme : pourtant, seule la dernière valeur sera retenue, bien qu'elle soit plus petite.

\section{III.2.5. La méthode POT et le modèle Poisson-GPD}

La dernière méthode est reconnue comme la plus appropriée pour l'étude du comportement extrême d'une variable unique (voir par exemple [3]). Il s'agit de la méthode du renouvellement, plus connue sous son acronyme anglais POT (Peaks-Over-Threshold). Le principe est simple : il s'agit de fixer un seuil et de ne retenir que les pics des évènements dépassant ce seuil.

Contrairement à la méthode des maxima annuels, la taille de l'échantillon dépend du seuil, fixé arbitrairement. Il en est de même pour les résultats. En outre, il est nécessaire en pratique d'introduire dans le traitement de la série temporelle de départ des paramètres temporels qui permettent d'assurer l'indépendance des données, les durées minimale et maximale des évènements ainsi que la prise en compte d'éventuelles fluctuations autour du seuil au sein d'une même tempête. Ces paramètres dépendant fortement de la valeur du seuil, il convient donc d'être particulièrement vigilant au choix de ce dernier (voir par exemple [10]).

Considérons à nouveau un échantillon de $n$ variables indépendantes $\left(X_{1}, \ldots, X_{n}\right)$, distribuées suivant une distribution inconnue $F$, et considérons la variable $Y=X-u$, représentant le dépassement par $X$ d'un seuil $u$. La probabilité de non-dépassement de la variable $Y$, c'est-à-dire sa fonction de répartition, est donnée par :

$$
\operatorname{Pr}\{Y \leq y \mid X>u\}=\frac{F(u+y)-F(u)}{1-F(u)}
$$

Lorsque $u$ est suffisamment grand, cette expression peut être approchée par la Distribution Généralisée de Pareto, ayant pour acronyme anglais GPD, et dont la fonction de répartition est donnée par :

$$
\begin{aligned}
& G_{Y, k, \sigma, \mu}(y)=1-\left(1+k \frac{y-\mu}{\sigma}\right)^{-\frac{1}{k}} \text { si } k \neq 0 \\
& G_{Y, \sigma, \mu}(y)=1-\exp \left(-\frac{y-\mu}{\sigma}\right) \text { si } k=0
\end{aligned}
$$

On retrouve les trois paramètres de forme $k$, d'échelle $\sigma$ et de localisation $\mu$. Lorsque $k>0$, le point terminal (c'està-dire la valeur $y_{\max }$ pour laquelle la probabilité de dépassement est nulle) de la distribution est infini ; lorsque $k=0$ la GPD n'est autre que la loi exponentielle; et lorsque $k<0$, la loi admet un point terminal fini qui vaut $\mu-\sigma / k$.

La loi GPD est donc une loi asymptotique qui donne une bonne approximation de la loi des excès de la distribution inconnue $F$ (équation 6) lorsque $u$ tend vers le point terminal, fini ou infini, de $G$. L'hypothèse complémentaire étant faite que le nombre de tempêtes dépassant le seuil suit un processus de Poisson, le modèle obtenu est appelé modèle Poisson-GPD.

Cunnane (1973) [4] puis Madsen et al. (1997a) [11] ont montré que l'approche POT sera préférable aux maxima annuels dans la plupart des cas, si le nombre moyen de dépassements du seuil par an est supérieur à 1,65. De plus, le Groupe de Travail sur les Statistiques des Houles Extrêmes a souligné la pertinence de l'approche POT pour prendre en compte la forte variabilité interannuelle des évènements de tempête.

\section{III.2.6. Autres modèles}

On présente uniquement ici les modèles les plus classiques (GEV, GPD), mais ceux-ci peuvent être étendus. Par exemple, les présents auteurs [13] utilisent une approche multi-distributions pour les échantillons POT (d'autres lois que la GPD sont examinées).

D'autres approches peuvent aussi être utilisées. Ainsi, aux Pays-Bas, van Gelder (1996) [24] applique des analyses bayésiennes aux échantillons POT ou de type maxima annuels, et Proske et van Gelder (2006) [16] s'appuie sur le modèle de Grey, qui repose sur une description mathématique de l'incertitude.

\section{III.3. Les méthodes indirectes}

\section{III.3.1. L'approche anglaise : la Joint Probability Method (JPM) et ses variantes}

Pugh \& Vassie (1978) [17] ont défini la méthode des probabilités combinées (JPM). Toutes les mesures (généralement horaires, mais également, pour les mesures les plus récentes, toutes les 15 minutes) sont utilisées. Les observations de surcote et de marée astronomique sont séparées. Puis un histogramme est réalisé pour chaque composante, avec un intervalle de discrétisation de $0,1 \mathrm{~m}$.

La densité de probabilité de la marée astronomique s'obtient aujourd'hui facilement pour la plupart des ports. Il suffit de disposer d'un cycle long de 18,6 ans de mesures pour obtenir la quasi-intégralité de la densité de probabilité recherchée. Pour les surcotes, on est limité par la durée des mesures.

Une convolution discrète est ensuite réalisée pour obtenir la probabilité combinée marée + surcote.

Une première hypothèse de la JPM est donc de considérer que les valeurs de surcotes horaires sont indépendantes. Un autre point important est que les surcotes ne sont pas extrapolées : on fait l'hypothèse que les observations sont représentatives des évènements extrêmes à venir. Par cette méthode, la probabilité est nulle de dépasser le niveau qui additionne la plus haute marée astronomique et la plus haute surcote observée. On retrouve là les estimations plus anciennes, en vigueur avant l'introduction de ces méthodes statistiques.

Plusieurs améliorations de cette méthode ont été proposées, dont la RJPM (Revised Joint Probability Method) et 
la SRJPM (Spatial Revised Joint Probability Method). La RJPM [22] prend en compte les deux inconvénients majeurs de la JPM cités ci-dessus.

En premier lieu, les surcotes sont extrapolées, c'est-à-dire qu'une véritable analyse extrême est réalisée. Le modèle retenu est celui des $r$-plus grandes valeurs, restreinte à la distribution de Gumbel ajustée par l'estimateur du maximum de vraisemblance (EMV). Cette approche, couplée à un traitement POT de la série temporelle, permet de réaliser l'extrapolation sur des données indépendantes. $r$ est choisi de façon à obtenir, en moyenne, environ cinq évènements par an. La distribution $H$ des surcotes extrêmes (c'est-à-dire celle des maxima des $K$ années de l'échantillon) est ensuite transposée en une distribution $F_{s}$ des surcotes horaires extrêmes en tenant compte de la dépendance entre les données horaires par l'emploi d'un indice de dépendance $\theta_{s}$ :

$$
F_{s}(x)=H^{\frac{1}{N K \theta_{s}}}(x)
$$

$N$ est le nombre d'observations horaires dans une année (8766) et $K$ la durée de la série temporelle, en années ( $n=N K$ est donc le nombre total de données horaires de la série temporelle). $\theta_{s}$ est compris entre 0 et 1 (cas de données indépendantes). On peut interpréter $1 / \theta_{s}$ comme la durée moyenne d'une tempête. On obtient alors facilement la densité correspondante $f_{s}$, ce qui permet d'affecter une probabilité aux surcotes supérieures au maximum observé, puis on la raccorde à la densité des surcotes observées. Une convolution à la densité des hauteurs de marée astronomique permet d'obtenir les niveaux d'eau.

La SRJPM, quant à elle, prend également en compte l'information relative à la variation spatiale de la marée et des surcotes, ce qui lui permet d'incorporer tous les types de données disponibles $[6,7]$.

Haigh et al. (2010) [8], ont mené des comparaisons et des analyses de sensibilité sur deux méthodes directes (la méthode des maxima annuels et la méthode des $r$-plus grandes valeurs) et deux méthodes indirectes (la JPM et la RJPM). Pour cela ils ont étudié 18 sites en Manche, dont 9 du côté français. Ils ont enfin comparé les résultats donnés par ces quatre méthodes d'analyse à ceux de la SRJPM. Leur conclusion est de favoriser la RJPM.

\section{III.3.2. Les calibrations de Pirazzoli et Tomasin}

Pirazzoli et Tomasin (2007) [15] et Tomasin et Pirazzoli (2008) [23] proposent différentes calibrations empiriques de la JPM, car selon eux, elle surestime les valeurs de retour.

La première calibration consiste à caler, par un coefficient approprié $C_{c}$, les résultats de la JPM de façon à ce que le niveau extrême de période de retour égale à la durée des observations $(T=K)$ corresponde au plus haut niveau mesuré.

Pour expliquer physiquement ces corrections aboutissant à faire baisser les résultats, ils invoquent deux formes de dépendance : l'interaction surcote-marée et la saisonnalité. Dans le premier cas, ils observent que les surcotes extrêmes apparaissent plus rarement qu'attendu lors des hauts niveaux de marée. Par l'emploi d'un coefficient ad hoc, noté $C_{99,9}$ une nouvelle calibration est ainsi proposée. Dans le deuxième cas (qui recoupe d'ailleurs le premier), il est observé, comme l'on peut s'y attendre, que les plus fortes surcotes ont généralement lieu l'hiver alors que les plus grandes marées ont lieu lors des équinoxes. Une dernière calibration basée sur la correspondance temporelle entre fortes marées et fortes surcotes est alors proposée, à l'aide d'un coefficient $C_{M M}$.
Les auteurs favorisent la première calibration. Au final, les méthodes de Pirazzoli et Tomasin sont donc des calibrations empiriques visant à prendre en compte les phénomènes de dépendance entre la marée astronomique et les surcotes. En revanche, ces dernières ne peuvent dépasser le maximum observé (pas d'extrapolation). Pourtant, particulièrement pour les séries temporelles de durée faible ou moyenne, il n'y a a priori aucune raison que, systématiquement, le plus fort évènement observé ait une période de retour égale à la durée de la série temporelle.

\section{III.3.3. La méthode Simon (SHOM / CETMEF)}

La méthode Simon (1994) [19] est également une variation de la JPM : elle consiste en la combinaison des lois de probabilité, non plus des observations horaires, mais des hauteurs de pleine mer prédites et des valeurs des surcotes observées à ces mêmes pleines mer. Les surcotes observées (définies précisément comme la différence entre le niveau maximum observé autour de l'heure théorique de pleine mer et le niveau prédit de pleine mer) sont traitées à l'aide d'un modèle d'extrapolation, en l'occurrence la distribution de Gumbel. Il s'agit donc d'une approche particulière, différente des constitutions classiques de l'échantillon des surcotes présentées en III.2. Pour ses promoteurs, elle possède deux grands avantages : d'une part, Simon (1994) [19] a mis en évidence l'indépendance de la marée et des surcotes pour les évènements de pleine mer, et d'autre part, cette restriction au plus haut niveau observé permet d'éviter des erreurs parfois importantes dues à un décalage temporel causé soit par une erreur dans l'estimation des horaires de marée, soit par un horaire imprécis de relevé des mesures de niveau.

Dans les ports secondaires où peu de données sont disponibles, l'idée est de profiter des observations simultanées avec un port de référence proche, d'estimer la corrélation entre les surcotes pour ces deux points et d'interpoler les données manquantes en gardant la même corrélation.

\section{LES RÉSULTATS DES ÉTUDES PAR FACADE}

\section{IV.1. Manche et Mer du Nord}

L'étude du SHOM/CETMEF réalisée en 1994 [19,21] a été mise à jour en 2008 (sous forme de CD-ROM) avec des données marégraphiques courant jusqu'en 2004. Les résultats ne sont disponibles que pour la Mer du Nord, la Manche et l'Atlantique. Une nouvelle mise à jour est à l'étude, avec une extension des données marégraphiques ; un rapport complet doit sortir au milieu de l'année 2011. Tomasin et Pirazzoli (2008) [23] fournissent les niveaux extrêmes pour 10,50 et 100 ans pour la méthode JPM corrigée par le coefficient $C_{c}$. Nous avons transposé les résultats dans le référentiel IGN 69 pour permettre une comparaison avec la méthode Simon. Enfin, les études anglaises [8] sont également disponibles pour huit ports de cette façade. Le tableau 1 récapitule les niveaux centennaux dans le référentiel IGN 69. Entre parenthèses, on précise la durée effective (en années équivalentes) des séries utilisées.

Une première observation évidente est que les niveaux de la méthode Simon sont toujours supérieurs à ceux de la RJPM, mais restent cependant assez proches : généralement moins de $0,1 \mathrm{~m}$, et toujours moins de $0,15 \mathrm{~m}$. Les résultats de Pirazzoli et Tomasin sont fluctuants autour de ces deux méthodes, mais sont souvent nettement inférieurs. 
Tableau 1 : Niveaux d'eau centennaux pour 10 ports de la Manche et de la Mer du Nord (référence IGN 69) et durée de la série utilisée (années équivalentes).

\begin{tabular}{|l|c|c|c|c|}
\hline \multicolumn{1}{|c|}{ Port } & $\begin{array}{c}\text { Simon } \\
\text { Etude 2008 }\end{array}$ & $\begin{array}{c}\text { Haigh et al. } \\
\text { RJPM }\end{array}$ & $\begin{array}{c}\text { Pirazzoli/Tomasin } \\
\text { JPM corrigée }\end{array}$ & Simon / RJPM \\
\hline Dunkerque & $4,72(35,7)$ & $4,66(48)$ & $4,89(33,9)$ & $+0,06$ \\
\hline Calais & $4,98(10,2)$ & $4,94(40)$ & $5,00(26,7)$ & $+0,04$ \\
\hline Boulogne & $5,84(17,9)$ & $5,71(30)$ & $5,63(17,4)$ & $+0,13$ \\
\hline Dieppe & $6,31(32,6)$ & - & $6,21(31,4)$ & \\
\hline Le Havre & $4,89(30,2)$ & $4,87(42)$ & $4,77(32,8)$ & $+0,02$ \\
\hline Cherbourg & $4,21(28,7)$ & $4,17(36)$ & $4,01(28,6)$ & $+0,04$ \\
\hline Saint-Malo & $7,61(9,3)$ & - & $7,48(46,3)$ & \\
\hline Roscoff & $5,41(27,8)$ & $5,34(33)$ & $5,33(20,2)$ & $+0,07$ \\
\hline Le Conquet & $4,64(32,1)$ & $4,50(36)$ & $4,47(30,4)$ & $+0,14$ \\
\hline Brest & $4,74(131,4)$ & $4,61(99)$ & $4,51(129,7)$ & $+0,13$ \\
\hline
\end{tabular}

\section{IV.2. La façade atlantique}

Sur la façade atlantique, on dispose principalement de l'étude SHOM/CETMEF et des travaux de Pirazzoli et Tomasin (2007) [15]. Les niveaux centennaux sont présentés dans le tableau 2. Là aussi, les estimations de Pirazzoli et Tomasin sont nettement en dessous de la méthode Simon.

\section{IV.3. Le littoral méditerranéen}

Peu d'études de niveaux extrêmes ont été effectuées le long du littoral méditerranéen, qui se caractérise par une prédominance de la composante de surcote. Bien que les méthodes indirectes restent évidemment valables, les méthodes directes peuvent y être utilisées avec profit au vu de leur simplicité.

Les surcotes peuvent être particulièrement importantes dans le Golfe du Lion, du fait de l'étendue du plateau continental. Le caractère accore de la côte entre Marseille et Nice, en revanche, fait que les surcotes y sont moins prononcées.

Dans le cadre du projet DISCOBOLE, Pirazzoli (2006) [14] a appliqué ses méthodes sur sept ports de la façade méditerranéenne et a recommandé les valeurs obtenues par sa calibration par le coefficient $C_{c}$ (ou, en cas d'impossibilité, par la JPM seule). De son côté, le CETE-Méditerranée (2010) [2] a effectué pour le compte de la DREAL Languedoc-Roussillon un travail très important de nettoyage des données marégraphiques de quatre stations de la région pour numériser les anciens marégrammes analogiques et corriger les valeurs aberrantes. Les niveaux horaires extrêmes ont ensuite été calculés par des méthodes directes (maxima annuels avec distribution de Gumbel et méthode POT avec distribution exponentielle). On donne ici les résultats pour les maxima annuels, jugés plus fiables par les auteurs. Les résultats sont présentés dans le tableau 3 .

\section{PISTES D'AMÉLIORATION ET PERSPECTIVES}

\section{V.1. Approches spatiales}

Les méthodes spatiales permettent soit d'augmenter la quantité d'information en entrée du calcul (prise en compte de mesures plus seulement au niveau local mais au niveau régional), soit d'augmenter les résultats en sortie en interpo-

Tableau 2 : Niveaux d'eau centennaux pour 11 ports de la façade Atlantique (référence IGN 69) et durée de la série utilisée (années équivalentes).

\begin{tabular}{|l|c|c|}
\hline \multicolumn{1}{|c|}{ Port } & Méthode Simon 2008 & $\begin{array}{c}\text { Pirazzoli / Tomasin - JPM } \\
\text { corrigée }\end{array}$ \\
\hline Concarneau & - & $3,65(3,3)$ \\
\hline Port-Tudy & $3,80(28,7)$ & $3,47(20,3)$ \\
\hline Le Crouesty & - & $3,53(1,3)$ \\
\hline Saint-Nazaire & - & $3,49(21)$ \\
\hline Saint-Gildas & $3,80(24,4)$ & $3,32(23,3)$ \\
\hline Les Sables-d'Olonne & $3,66(15,3)$ & $3,55(13,9)$ \\
\hline La Rochelle & $3,96(21,7)$ & $3,81(18,6)$ \\
\hline Port-Bloc - Pointe de Grave & $3,83(12,4)$ & $3,60(14,9)$ \\
\hline Arcachon & - & $3,26(4,1)$ \\
\hline Boucau & - & $3,05(27,4)$ \\
\hline Saint-Jean-de-Luz & $3,08(33,2)$ & $3,07(11,4)$ \\
\hline
\end{tabular}


Tableau 3 : Niveaux d'eau centennaux pour 9 ports de la façade Atlantique (référence IGN 69) et durée de la série utilisée (années équivalentes).

\begin{tabular}{|l|c|c|}
\hline \multicolumn{1}{|c|}{ Port } & Pirazzoli - DISCOBOLE (2006) & CETE-Méditerranée (2010) \\
\hline Banyuls-sur-Mer & $0,82(5,6)$ & - \\
\hline Port-Vendres & $0,90(13,7)$ & $0,84(21)$ \\
\hline Sète (station SHOM) & $1,06(5,7)$ & $1,08(37)$ \\
\hline Le Grau-de-la-Dent & - & $1,04(34)$ \\
\hline Thau & - & $0,91(19)$ \\
\hline Marseille & $1,17(82,9)$ & \\
\hline Toulon & $0,73(18)$ & \\
\hline Monaco & $0,69(9,8)$ & \\
\hline Nice & $0,63(6,7)$ & \\
\hline
\end{tabular}

lant les résultats d'un port à l'autre pour aboutir à des cartes de niveaux extrêmes, qui ne soient plus limitées aux ports de référence pourvus d'un marégraphe sur une période suffisamment longue. Ces interpolations doivent cependant être réalisées avec précaution, surtout lorsque la topographie et/ ou la bathymétrie locales sont complexes. Elles ne peuvent en particulier s'appliquer aux havres, bassins, estuaires et lagunes.

Les études SHOM / CETMEF utilisent des mesures de courte durée pour calibrer l'interpolation permettant de calculer la marée astronomique puis les niveaux sur l'ensemble des façades étudiées. Les équipes anglaises (Dixon et Tawn, 1995, 1997) [6,7] ont construit la SRJPM (Spatial Revised $J P M)$ pour traiter l'ensemble des données disponibles (mesures horaires, maxima annuels, reconstitutions numériques). On peut ensuite calculer les niveaux sur n'importe quel point de la façade étudiée.

Bernardara et al. (2011) [1], du Laboratoire National d'Hydraulique et Environnement (LNHE) d'EDF, ont appliqué aux surcotes de pleine mer la méthode RFA, pour Regional Frequency Analysis. Cette méthode, introduite par Dalrymple (1960) [5] et détaillée par Hosking et Wallis (1997) [9], repose sur l'hypothèse qu'à l'échelle régionale, toutes les données de la grandeur considérée (ici les surcotes) obéissent à une même distribution (seul le paramètre d'échelle est propre à chaque site). L'approche POT étant utilisée pour bâtir les échantillons sur chaque site local, la loi choisie est une GPD. Des tests d'homogénéité basés sur les L-moments permettent de définir des régions homogènes auxquelles appliquer l'analyse. Bernardara et al. ont considéré l'ensemble des façades Atlantique, Manche et Mer du Nord comme une région homogène (soumise aux dépressions atlantiques) et en ont dérivé les surcotes millennales pour dix-huit ports, de Dunkerque à Saint-Jean-de-Luz.

\section{V.2. Homogénéisation des surcotes}

Une autre piste de travail concerne le pré-traitement de l'échantillon des surcotes. Les présents auteurs [13] ont rappelé l'importance de séparer les échantillons de vagues en populations homogènes distinctes pour une bonne analyse statistique destinée à déterminer les vagues extrêmes. Or, cette même recommandation peut s'appliquer aux surcotes / décotes météorologiques. Si deux régimes de vent clairement différents peuvent générer les plus grandes surcotes sur un site donné, il sera nécessaire de les étudier séparément. La difficulté réside dans le fait que les mesures de maré- graphes n'offrent aucune information autre que le niveau, contrairement aux bouées donnant, en plus de la hauteur des vagues, leur direction et leur période, caractéristiques permettant d'identifier les différentes populations (houle, mer de vent...). Une analyse tempête par tempête est alors nécessaire, ce qui peut être lent et fastidieux. À l'avenir, cependant, on peut penser qu'avec l'intégration des calculs de surcotes dans les modèles numériques météo-océanographiques, l'homogénéisation des surcotes sera plus aisée. Notons par ailleurs que, cette approche diminuant la taille des échantillons auxquels on applique l'analyse statistique, il vaut mieux ne l'appliquer que lorsque les durées d'enregistrement disponibles sont suffisamment grandes.

\section{CONCLUSIONS}

La méthodologie de détermination des niveaux extrêmes dépend donc du poids relatif des deux composantes déterministe et stochastique. Une méthode indirecte est indispensable lorsque la composante stochastique n'est pas prédominante. Deux grandes méthodes sont actuellement utilisées : la méthode Simon basée sur les hauteurs de pleine mer et les développements de la méthode JPM utilisant toutes les données mesurées. La comparaison montre qu'elles donnent toutes deux des résultats très similaires en Manche et Mer du Nord. Pour le dimensionnement des ouvrages de protection et la définition des zones à risque, les différentes calibrations de Pirazzoli et Tomasin ne semblent pas suffisamment étayées pour justifier le risque de baisser souvent significativement les niveaux de référence. Les deux façades Manche et Atlantique sont largement étudiées. En revanche, le littoral méditerranéen n'a fait l'objet d'études approfondies que depuis peu, alors que le risque de submersions doit y être pris au sérieux. Enfin, les méthodologies peuvent être encore améliorées par une bonne prise en compte de l'information disponible au niveau régional ainsi que par une homogénéisation des surcotes seules.

\section{BIBLIOGRAPHIE}

[1] Bernardara P., Andreewsky M., Benoit M. (2011) Application of regional frequency analysis to the estimation of extreme storm surges. Journal of Geophysical Research. 116, C02008 
[2] Cete-Mediterranee (2010) - Traitement des series marégraphiques du Golfe du Lion - Extraction et étude statistique des évènements de reference. Rapport établi pour le compte de la DREAL Languedoc-Roussillon

[3] Coles S. (2001) - An Introduction to Statistical Modeling of Extreme Values. Springer ed., London

[4] Cunnane C. (1973) - A particular comparison of annual maxima and partial duration series methods of flood frequency prediction. Water Resour. Res. 391216

[5] Dalrymple T. (1960) - Flood frequency analysis. U.S. Geol. Surv. Water Supply Pap. 1543-A

[6] Dixon M.J., TAWn J.A. (1995) — Extreme sea-levels at the UK A-class sites : optimal site-by-site analysis and spatial analyses for the East Coast. (http ://www.pol.ac.uk/ntslf/pdf/ id72.pdf) POL Internal. 72

[7] Dixon M.J., TAwn J.A. (1997) - Estimates of extreme sea conditions - final report, spatial analysis for the UK coast. http ://www.pol.ac.uk/ntslf/pdf/id112.pdf) POL Internal. 112

[8] Haigh I.D., Nicholls R., Wells N. (2010) — A comparison of the main methods for estimating probabilities of extreme still water levels. Coastal Engineering. 57 838-849

[9] Hosking J.R.M., Wallis J.R. (1997) - Regional Frequency Analysis : an approach based on L-moments. Cambridge Univ. Press, Cambridge, U.K.

[10] LANG M.,OuARda T.B.M.J., BobÉE B. (1999) — Towards operational guidelines for over-threshold modelling. J. Hydrol. 225 103-117

[11] Madsen H., Rasmussen P.F., Rosbjerg D. (1997) Comparison of annual maximum series and partial duration series for modeling extreme hydrologic events, 1. At-site modeling. Water Eesour. Res. 33 747-757

[12] Mathiesen M., Goda Y., Hawkes P.J., Mansard E., Martin M.J., Peltier E., Thompson E.F., Van Vledder G. (1994) Recommended practice for extreme wave analysis. J. Hydraul. Res. 32(6) 803-814

[13] Mazas F., Намм L. (2011) - A multi-distribution approach to POT methods for determining extreme wave heights. Coastal Engineering
[14] Pirazzoli P.A. (2006) - Projet DISCOBOLE. Contribution à la tâche 5 : calcul de hauteur des niveaux d'eau extrêmes sur le littoral français. Disponible sur

[15] Pirazzoli P.A., Tomasin A. (2007) - Estimation of return periods for extreme sea levels : a simplified empirical correction of the joint probabilities method with examples from the French Atlantic coast and three ports in the southwest of the UK. Ocean Dynamics. 57 91-107

[16] Proske D., Van Gelder P.H.A.J.M. (2006) - Analysis about extreme water levels along the Dutch north-sea using Grey models : preliminary analysis. Safety and Reliability for Managing Risk, Guedes Soares \& Zio (eds)

[17] Pugh D.T., VAssie J.M. (1978) — Extreme sea levels from tide and surge probability. Coastal Engineering. 911-930

[18] Pugh D.T., Vassie J.M. (1980) - Application of the joint probability method for extreme sea level computations. Proc. Instn Civ Engrs, Part 2. 69 959-975

[19] Simon B. (1994) — Les niveaux marins extrêmes le long des côtes de France et leur évolution. Rapport SHOM 001/94

[20] Simon B. (2007) - La marée océanique côtière.Co-édition de l'Institut Océanographique et du SHOM, ISBN 978-2-90358132-9

[21] Shom, Cetmef (2008) - Statistique des niveaux marins extrêmes de pleine mer : Manche et Atlantique

[22] TAWn J.A., VAssie J.M. (1989) - Extreme sea levels : the joint probabilities method revisited and revised. Proc. Instn Civ Engrs, Part 2. 87 429-442

[23] Tomasin A., Pirazzoli P.A. (2008) - Extreme sea levels in the English Channel : calibration of the joint probability method. Journal of Coastal Research. 24(4C) 1-13

[24] Van Gelder P.H.A.J.M. (1996) - A new statistical model for extreme water levels along the Dutch coast. Stochastics Hydraulic'96 : Proceedings of the International Symposium on Stochastic Hydraulics, MacKay 